\title{
Association between mitochondrial DNA and cognitive impairment in schizophrenia: study protocol for a Mexican population
}

This article was published in the following Dove Press journal:

Neuropsychiatric Disease and Treatment

\section{Dulce Dajheanne García-de} la Cruz, ${ }^{1,2}$ Isela Esther Juárez-Rojop,' Carlos Alfonso Tovilla-Zárate, ${ }^{3}$ José Jaime Martínez-Magaña, I,4 Alma Delia Genis-Mendoza, ${ }^{4,5}$ Humberto Nicolini, ${ }^{4}$ Thelma Beatriz González-Castro, 1,6 Crystell Guadalupe Guzmán-Priego,' Nancy Adanelly López-Martínez, ,2 Javier Antonio Hernández-Cisneros,' Francisco Caballero-Prado ${ }^{7}$

'División Académica de Ciencias de la Salud, Universidad Juárez Autónoma de Tabasco, Villahermosa, Tabasco, Mexico; ${ }^{2}$ Investigación y Enseñanza, Hospital Regional de Alta Especialidad de Salud Mental, Villahermosa, Tabasco, Mexico; ${ }^{3}$ División Académica Multidisciplinaria de Comalcalco, Universidad Juárez Autónoma de Tabasco, Villahermosa, Tabasco, Mexico; ${ }^{4}$ Laboratorio de Enfermedades Psiquiátricas y Neurodegenerativas, Instituto Nacional de Medicina Genómica, Mexico City, Mexico; ${ }^{5}$ Hospital Psiquiátrico Infantil "Dr. Juan N. Navarro", Mexico City, Mexico; ${ }^{6}$ División Académica Multidisciplinaria de Jalpa de Méndez, Universidad Juárez Autónoma de Tabasco, Jalpa de Méndez, Tabasco, Mexico; ${ }^{7}$ Servicio de Salud Mental, Hospital Regional de Alta Especialidad "Dr. Juan Graham Casasús", Villahermosa, Tabasco, Mexico

Correspondence: Alma Delia GenisMendoza

Instituto Nacional de Medicina Genómica (INMEGEN), Servicios de Atención Psiquiátrica (SAP), Periférico Sur 4809, Arenal Tepepan, Mexico City I46I0, Mexico

Tel +52 5553501900

Email adgenis@inmegen.gob.mx
Background: Circulating cell-free mitochondrial DNA (cf-mtDNA) fragments in blood plasma have been reported in patients with schizophrenia (SZ). Although the relationship of cf-mtDNA to the cognitive status of patients with SZ has not yet been explored, it is known that cognitive impairment in SZ compromises the functional and social capacity of these patients and diminishes their quality of life. In this sense, the assessment of the severity of cognitive impairment in a Mexican population with SZ and its association with cf-mtDNA levels in blood plasma may provide the possibility of using cf-mtDNA as a biomarker to determine the status of the disease and the possible ensuing changes over time.

Methods: Subjects for a case-control study will be recruited. cf-mtDNA obtained from blood plasma will be quantified by real-time polymerase chain reaction, using melting curve technology with SYBR green as amplification marker. Patients with SZ will be grouped into those with severe, mild, and no cognitive impairment according to Montreal Cognitive Assessment scale scores, to determine differences between cognitive performance and cfmtDNA levels in blood plasma.

Ethics and communication: This study has been approved by the ethics and investigation committees of the High Specialty Regional Hospital of Mental Health (Hospital Regional de Alta Especialidad de Salud Mental); project No. HRAESM/DG/RP/1128/2018. We plan to communicate our research findings in scientific conferences and in peer-reviewed journals. Conclusion: It is known that cognitive dysfunction provokes negative effects in an SZ patient's life. This project aims to provide better knowledge about the role of cf-mtDNA in the pathogenesis of cognitive impairment in SZ, as an attempt to achieve improvements to the existing treatments, thereby helping to prevent major cognitive deterioration.

Keywords: schizophrenia, cognitive impairment, mitochondrial dysfunction, mitochondrial DNA, neurodegeneration

\section{Introduction}

Among the diversity of clinical symptoms exhibited by patients with schizophrenia (SZ), cognitive impairment worsens with the onset of the first psychotic episode. This symptom has been reported to start on earlier phases of the disease as abnormalities in social behavior and academic performance in childhood or early adolescence. Hence, the normal development of these patients will be prevented and will affect their everyday functioning in adulthood. ${ }^{1-5}$ In this regard, several studies have estimated a low remission rate of symptoms, approximately $13.5 \%$ as upper limit. ${ }^{6}$ On the other hand, cognitive impairment is associated with a bad life prognosis, which compromises 
the functional and social capacity of these subjects and diminishes their quality of life. As a result, several studies with multivariate approaches have been undertaken to get a better understanding of the role of cognitive impairment in SZ. Among the multiple factors involved, there is some evidence of differences between mtDNA copy number from whole blood in patients with SZ compared to healthy subjects. $^{7,8}$ Moreover, circulating cell-free mtDNA (cfmtDNA) fragments in blood plasma have also been reported in patients with mood disorders and SZ. ${ }^{9}$ However, their association with the cognitive status of patients with SZ has not yet been explored. Quantification of the number of copies of cf-mtDNA in plasma of these patients according to their cognitive status may provide the possibility of using cfmtDNA as a biomarker for a better assessment of the status of the disease and the possible changes that will ensue over time. In addition, better strategies of treatment may be promoted to prevent progressive deterioration of these patients.

\section{Objective}

Our aim is to assess the severity of cognitive impairment in a Mexican population with $\mathrm{SZ}$ and to evaluate its association with cf-mtDNA levels in blood plasma, so that cf-mtDNA could be used as a possible biomarker to prevent a major deterioration of schizophrenic patients.

\section{Methods and analysis}

\section{Participants}

Patients with the previous diagnosis of SZ established by a psychiatrist in accordance with the DSM-5 instrument will be recruited from the High Specialty Regional Hospital of Mental Health in the city of Villahermosa, Tabasco, Mexico. These subjects will conform the case group $(n=100)$. Alternatively, a control group $(n=100)$ will be integrated by healthy subjects with no clinical history of psychiatric, neurological, or clinical disease and will be recruited from the Blood Donor Center of the General Hospital of Comalcalco, in Comalcalco, Tabasco. Subjects included in both groups will be matched by gender and age (range of 1860 years of age). ${ }^{10}$ Given that medication is known to affect cognition, only patients taking no medication will be included. Patients with vascular risk factors such as type 2 diabetes or coronary heart diseases, as well as subjects with acute psychosis, psychotic disorder of toxic origin, neurovascular disease, dementia or moderate to severe brain injury, analphabets, or persons with any intellectual or physical incapacity will be excluded from the study.

\section{Ethics and reach out}

All subjects who decide to participate in the study should declare their acceptance in a written informed consent. The study will be performed according to the Helsinki Declaration (59th General Assembly, Seoul, Korea, October, 2008). This study has been approved by the ethics and research committees of the High Specialty Regional Hospital of Mental Health, project No. HRAESM/DG/RP/1128/2018. We plan to communicate our research findings in scientific conferences and peerreviewed journals.

\section{Assessments: cognitive impairment}

Impairments in executive, visual and spatial functions will be measured with the Montreal Cognitive Assessment scale (MoCA); scores of 26 points or higher represent normal cognitive functioning. One point will be added when the academic level is less than 12 years, in agreement with other authors. ${ }^{11}$

\section{Premorbid adjustment}

The Premorbid Adjustment Scale (PAS) will be used to assess academic and social performance in our subjects prior to the onset of SZ. This scale evaluates five domains - sociability and withdrawal, peer relationships, scholar performance, scholar adaptation, and socio-sexual function -, as well as four distinct periods of a patient's life _- childhood, early adolescence, late adolescence, and adulthood. The average of the total PAS varies from 0 to 1 ; lower scores represent better functioning of patients. ${ }^{12,13}$

\section{Positive, negative, cognitive, anxiety/ depression, and excitability symptoms}

The five-factor positive and negative syndrome scale (PANSS; positive, negative, cognitive, anxiety/depression and excitability factors) will be applied only to the case group. This instrument contains 30 items; each one evaluates the intensity of symptoms with a possible score from 1 to 7 . The minimum total score is 30 and the maximum $210 .{ }^{14}$

\section{mtDNA extraction and quantification}

Whole blood from all the subjects recruited in the present study will be collected in EDTA tubes by venipuncture. Extraction of mtDNA from plasma samples will be performed using the Gentra Puregene kit (Qiagen, USA), in accordance with the conditions previously established by 
the provider. The isolated DNA will be eluted in $200 \mu \mathrm{L}$ Buffer AE and quantified using spectrophotometric analysis at 260/280 $\mathrm{nm}$ in a NanoDrop2000 equipment; an electrophoresis analysis will be used for assessing mtDNA quality and quantity. mtDNA will be quantified by the real-time polymerase chain reaction (TaqMan multiplex assay), using the melting curve technology with SYBR green as amplification marker. Fluorescence intensity will be measured with a PCR-rt equipment, Quanto Studio 7 (Termofisher). A standard curve will be created by a dilution series of the purified PCR product from a healthy control subject, who will not be taking part in the present study. Ribonuclease $\mathrm{P}$ amplicon will be used as the endogenous reference gene and the mitochondrially encoded $\mathrm{NADH}$, specifically, the Ubiquinone Oxidoreductase Core Subunit 2 (MT-ND2) gene will serve for quantification. The following primers will be used to amplify the mitochondrial encoded NADH, specifically, the Ubiquinone Oxidoreductase Core Subunit 2 (MT-ND2) gene: forward primer: CACACTCATCACAGCGCTAA and reverse primer: GGATTATGGATGCGGTTGCT, as reported by Lindqvist et al. ${ }^{15}$ Reaction conditions will be the following: a total volume of $25 \mu \mathrm{L}$ per reaction will contain $5 \mu \mathrm{L}$ of template, $1 \mu \mathrm{L}$ of each primer, $12 \mu \mathrm{L}$ SYBR MIX, and $6 \mu \mathrm{L}$ of nuclease-free water. As a quality control, the testing will be run once in triplicate reactions. Amplification conditions will be the following: an initial denaturation step at $95^{\circ} \mathrm{C}$ for $10 \mathrm{mins}, 5010$-s cycles at $95^{\circ} \mathrm{C}$ for denaturation, $10 \mathrm{~s}$ at $60^{\circ} \mathrm{C}$ as alignment step and an extension step of $11 \mathrm{~s}$ at $70^{\circ} \mathrm{C}$. Fluorescence intensity will be assessed using a continuous melting curve analysis from $60{ }^{\circ} \mathrm{C}$ to $97^{\circ} \mathrm{C}$.

\section{Statistical analysis}

Epidemiologic differences between the case and control groups will be analyzed with a chi-squared test $\left(\mathrm{X}^{2}\right)$ and Student's t-test for categorical and continuous variables, respectively, and represented as mean and standard deviation, and percentage values. According to MoCA scores ( $\geq 26$ are normal values), the case group will be regrouped into severe, mild, and no cognitive impairment. A one-factor ANOVA will be performed for the comparison of cf-mtDNA levels in blood plasma among the three MoCA-scored groups. A comparison of MoCA and PAS scores between genders will be performed to explore the influence of gender in cognitive performance. Pearson's correlation will be carried out for the assessment of the effect of clinical symptoms and premorbid adjustment (MoCA, PANSS, and PAS scores) on the differences in cf-mtDNA levels in the case group. . Due to the possible influence of the age of onset of the first psychotic episode in SZ pathology, we plan to classify patients according to this variable. A step by step logistic regression adjusted by age, gender, and BMI will evaluate the influence of clinical and socio-demographic variables as predictors of changes in cf-mtDNA levels in plasma. We used the G*Power software for the calculation of the sample size. Since this study will include 100 individuals in the study group, the power yielded a value of $82.52 \%$, using the $\mathrm{X}^{2}$ test and an effect size of 0.3. A $p$-value $<0.05$ will be considered significant. All data will be analyzed using the R program.

\section{Discussion}

In the last decades, the negative impact of cognitive dysfunction in SZ has been recognized, given its functional and social implications and its effect on the quality of life of individuals. ${ }^{4}$ Nowadays, the information about this association has been evaluated in various European and Asian populations. The findings of this protocol study will generate relevant data for a better understanding of this relationship in the Mexican population. Currently, no information related to this matter is available in this population. It is estimated that more than $75 \%$ of the patients with SZ manifest cognitive impairment. ${ }^{16}$ Moreover, cognitive dysfunction in SZ usually appears in early phases of the disease and may precede the first psychotic episode. ${ }^{3}$ The natural course of cognitive symptoms seems to start in childhood or adolescence. In general, $45 \%$ of the children who develop SZ are cognitively affected since the age of $7 .{ }^{17}$ Longitudinal studies have demonstrated that people who develop SZ show deficits in working memory, attention, language speed, and disturbances in social behavior. ${ }^{18}$ It is estimated that the rate of conversion to psychosis is $20-35 \%$ when cognitive disturbances are manifest. ${ }^{19}$ In addition, cognitive impairment in SZ is associated with structural brain alterations. ${ }^{20}$ Previously, it has been suggested that the central nervous system susceptibility to mitochondrial damage could be the leading cause of neurodegeneration observed in SZ. ${ }^{21}$ Mitochondrial dysfunction has been linked to structural, functional, and cognitive alterations in the brain. ${ }^{22,23}$ Also, recent findings suggest mitochondrial dysfunction and oxidative stress as triggers of mtDNA release and consequently the participation of mtDNA in neurodegeneration. ${ }^{24}$ Recent evidence has suggested the involvement of neural inflammation in the cognitive status of patients with $\mathrm{SZ}^{25}$ Furthermore, it 
has been observed that some antipsychotic drugs (ie, clozapine) are toxic to mitochondria and promote inflammation (in-vitro). ${ }^{26}$ They can cause a decrement in mtDNA copy numbers as shown in whole blood studies of psychotic patients. $^{7}$ Due to the previous evidence, studies consider important to control the influence of medication or have more eligible exclusion criteria in the sample studied.

Despite the scarce information on this matter, some studies have reported mtDNA levels of patients with SZ. $\mathrm{Li}$ et al, reported that patients with SZ exhibit lower mtDNA levels in whole blood samples than those of control subjects. ${ }^{8}$ A similar study described a reducing effect of clozapine and risperidone on mtDNA copy numbers. ${ }^{7}$ In this sense, we assume that the importance of mtDNA resides in its predisposition to the accumulation of mutations, capacity to trigger a pro-inflammatory state, and apoptosis (Figure 1). It is worthwhile to mention that these studies were focused only on the determination of mtDNA copy numbers, but not in their correlation to cognitive symptoms. With regard to the association of plasma cf-mtDNA with SZ, there is previous evidence that addresses this relation. Kageyama et al (2018) studied a population of 109 individuals with major depressive disorders (MDD) and also evaluated data from 28 patients with bipolar disorder, 17 with SZ, and 29 subjects as healthy controls in order to explore whether cf-mtDNA levels are linked to inflammatory processes with the purpose to suggest them as biomarkers of mood disorders. ${ }^{9}$ Derived from this investigation, they found that cf-mtDNA levels in blood plasma exhibit a nominal positive correlation with GM-CSF, IL-2, and IL-4 cytokines in patients with MDD, but no statistical association was proposed for SZ. In spite of the previous evidence, there is no research to address in a direct way the association between circulating cf-mtDNA and cognitive impairment in SZ. Therefore, the findings derived from the present protocol could provide valuable information to get a better understanding of the progress and treatment of the schizophrenic disorder.

On the other hand, a prodromal phase for SZ has been described. It entails the deterioration of cognitive function and precedes an emerging psychotic episode. ${ }^{27}$ Thus, interventions during this phase have been suggested as strategies

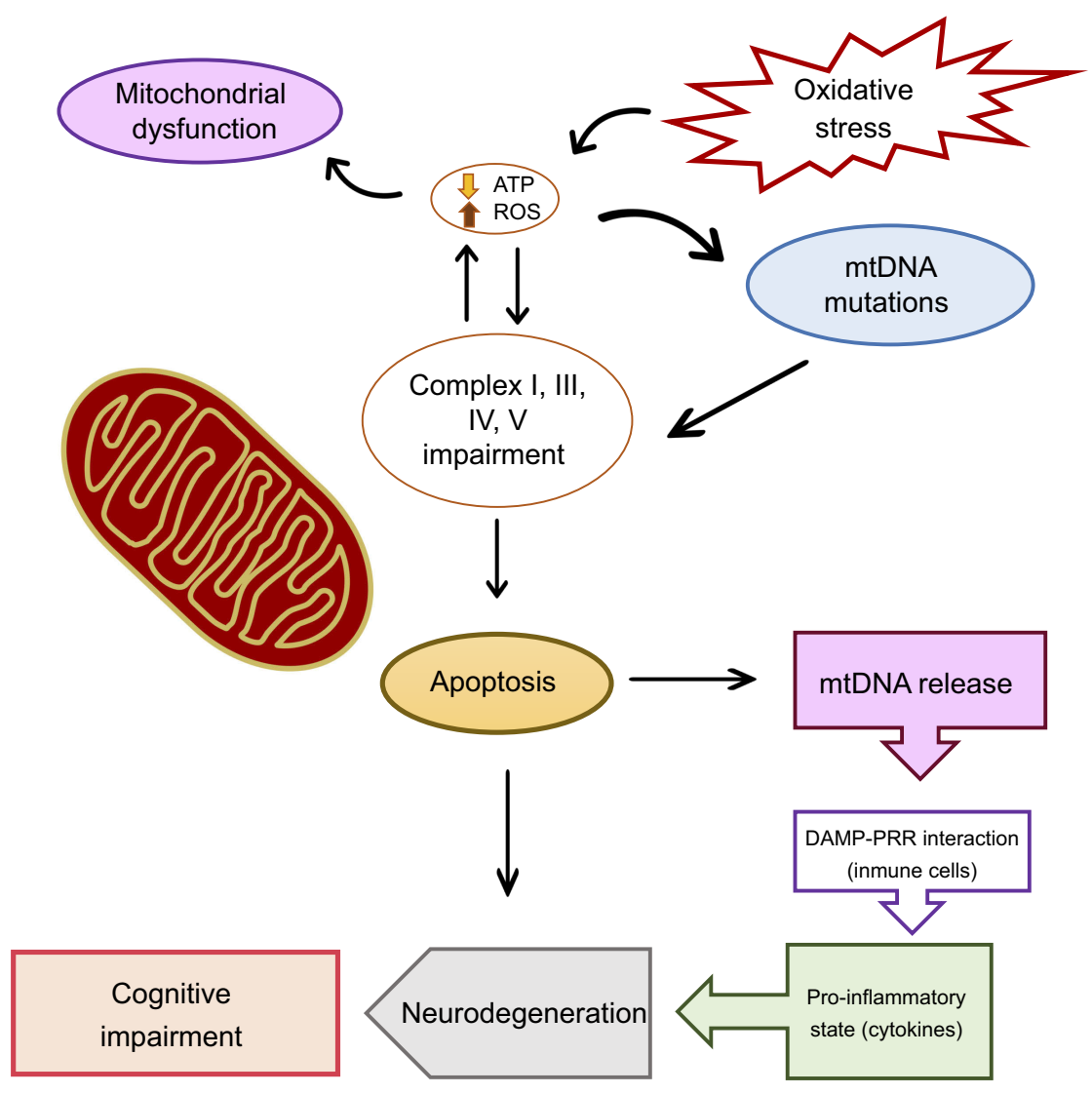

Figure I Mitochondrial DNA role in the pathogenesis of cognitive dysfunction in schizophrenia.

Abbreviations: mtDNA, mitochondrial DNA; ATP, adenosine triphosphate; ROS, reactive oxygen species; DAMP, damage associated molecular patterns; PRR, pattern recognition receptor. 
to reduce the transition to psychosis. ${ }^{28}$ Despite the emerging information in the last decades about cognitive dysfunction in SZ, there is no consensus for an adequate treatment. Hence, more studies in different populations are needed to gain a better understanding of cognitive impairment in SZ. In addition, psychiatrists do not count upon accessible diagnostic instruments to allow the prediction and detection of subtle cognitive changes in the early phases of the disease. Currently, a diagnosis for SZ is based on the duration and intensity of clinical symptoms, mainly when patients manifest their first psychotic symptoms.

Therefore, the aim of this protocol study is to measure the cognitive status and to quantify cf-mtDNA levels in blood plasma of schizophrenic patients in a Mexican population.

\section{Conclusion}

Circulating cf-mtDNA levels may serve as a potential biomarker to determine the cognitive status of patients with SZ. Thus, the measurement of cognitive symptoms in early phases of the disease along with the determination of cf-mtDNA levels in blood plasma may provide the possibility to prevent the worsening of cognitive functions, which in turn originates functional and social deficits.

\section{Acknowledgments}

Dulce Dajheanne García-de la Cruz is a CONACyT fellow (fellowship 890748) and received funding from the Programa de Fortalecimiento de la Calidad Educativa [Program for the Strengthening of Educational Quality], 2019; this study is part of her master degree.

\section{Disclosure}

The authors report no conflicts of interest in this work.

\section{References}

1. Jahshan C, Heaton RK, Golshan S, Cadenhead KS. Course of neurocognitive deficits in the prodrome and first episode of schizophrenia. Neuropsychology. 2010;24(1):109-120. doi:10.1037/a0016791

2. Shapiro DI, Marenco S, Spoor EH, Egan MF, Weinberger DR, Gold JM. The premorbid adjustment scale as a measure of developmental compromise in patients with schizophrenia and their healthy siblings. Schizophr Res. 2009;112(1-3):136-142. doi:10.1016/j.schres.2009. 04.007

3. Fisher M, Loewy R, Carter C, et al. Neuroplasticity-based auditory training via laptop computer improves cognition in young individuals with recent onset schizophrenia. Schizophr Bull. 2015;41(1):250-258. doi:10.1093/schbul/sbt232

4. Mantovani LM, Teixeira AL, Salgado JV. Functional capacity: a new framework for the assessment of everyday functioning in schizophrenia. Braz J Psychiatry. 2015;37(3):249-255. doi:10.1590/1516-4446-20141551
5. Strassnig MT, Raykov T, O'Gorman C, et al. Determinants of different aspects of everyday outcome in schizophrenia: the roles of negative symptoms, cognition, and functional capacity. Schizophr Res. 2015;165(1):76-82. doi:10.1016/j.schres.2015.03.033

6. Charlson FJ, Ferrari AJ, Santomauro DF, et al. Global epidemiology and burden of schizophrenia: findings from the Global Burden of Disease Study 2016. Schizophr Bull. 2018;44(6):1195-1203. doi:10.1093/schbul/sby058

7. Kumar P, Efstathopoulos P, Millischer V, et al. Mitochondrial DNA copy number is associated with psychosis severity and anti-psychotic treatment. Sci Rep. 2018;8(1):12743. doi:10.1038/s41598-018-31122-0

8. Li Z, Hu M, Zong X, et al. Association of telomere length and mitochondrial DNA copy number with risperidone treatment response in first-episode antipsychotic-naive schizophrenia. Sci Rep. 2015;5:18553. doi:10.1038/srep18553

9. Kageyama Y, Kasahara T, Kato M, et al. The relationship between circulating mitochondrial DNA and inflammatory cytokines in patients with major depression. J Affect Disord. 2018;233:15-20. doi:10.1016/j.jad.2017.06.001

10. Soleimani R, Shokrgozar S, Fallahi M, Kafi H, Kiani M. An investigation into the prevalence of cognitive impairment and the performance of older adults in Guilan province. J Med Life. 2018;11 (3):247-253. doi:10.25122/jml-2018-0017

11. Yang Z, Abdul Rashid NA, Quek YF, et al. Montreal cognitive assessment as a screening instrument for cognitive impairments in schizophrenia. Schizophr Res. 2018;199:58-63. doi:10.1016/j.schres.2018.03.008

12. Kilian S, Burns JK, Seedat S, et al. Factors moderating the relationship between childhood trauma and premorbid adjustment in firstepisode schizophrenia. PLoS One. 2017;12(1):e0170178. doi:10.1371/journal.pone.0170178

13. Cannon-Spoor HE, Potkin SG, Wyatt RJ. Measurement of premorbid adjustment in chronic schizophrenia. Schizophr Bull. 1982;8(3):470484. doi:10.1093/schbul/8.3.470

14. Emsley R, Rabinowitz J, Torreman M; Group R-I-EPGW. The factor structure for the Positive and Negative Syndrome Scale (PANSS) in recent-onset psychosis. Schizophr Res. 2003;61(1):47-57.

15. Lindqvist D, Wolkowitz OM, Picard M, et al. Circulating cell-free mitochondrial DNA, but not leukocyte mitochondrial DNA copy number, is elevated in major depressive disorder. Neuropsychopharmacology. 2018;43(7):1557-1564. doi:10.1038/ s41386-017-0001-9

16. Seidman LJ, Mirsky AF. Evolving notions of schizophrenia as a developmental neurocognitive disorder. J Int Neuropsychol Soc. 2017;23(9-10):881-892. doi:10.1017/S1355617717001114

17. Seidman LJ, Cherkerzian S, Goldstein JM, Agnew-Blais J, Tsuang MT, Buka SL. Neuropsychological performance and family history in children at age 7 who develop adult schizophrenia or bipolar psychosis in the New England Family Studies. Psychol Med. 2013;43 (1):119-131. doi:10.1017/S0033291712000773

18. Seidman LJ, Shapiro DI, Stone WS, et al. Association of neurocognition with transition to psychosis: baseline functioning in the second phase of the North American Prodrome Longitudinal Study. JAMA Psychiatry. 2016;73(12):1239-1248. doi:10.1001/ jamapsychiatry.2016.2479

19. Piskulic D, Liu L, Cadenhead KS, et al. Social cognition over time in individuals at clinical high risk for psychosis: findings from the NAPLS-2 cohort. Schizophr Res. 2016;171(1-3):176-181. doi:10.1016/j.schres.2016.01.017

20. Grassi S, Orsenigo G, Serati M, Caletti E, Altamura AC, Buoli M. Cognitive correlates of neuroimaging abnormalities in the onset of schizophrenia: a case report. World J Psychiatry. 2017;7(2):128-132. doi:10.5498/wjp.v7.i2.128

21. Olivero P, Lozano C, Sotomayor-Zárate R, et al. Proteostasis and mitochondrial role on psychiatric and neurodegenerative disorders: current perspectives. Neural Plast. 2018;2018:6798712. doi:10.1155/ $2018 / 6798712$ 
22. Rajasekaran A, Venkatasubramanian G, Berk M, Debnath M. Mitochondrial dysfunction in schizophrenia: pathways, mechanisms and implications. Neurosci Biobehav Rev. 2015;48:10-21. doi:10.1016/j. neubiorev.2014.11.005

23. Ben-Shachar D. Mitochondrial multifaceted dysfunction in schizophrenia; complex I as a possible pathological target. Schizophr Res. 2017;187:3-10. doi:10.1016/j.schres.2016.10.022

24. West AP. Mitochondrial dysfunction as a trigger of innate immune responses and inflammation. Toxicology. 2017;391:54-63. doi:10.1016/j.tox.2017.07.016

25. Johnsen E, Fathian F, Kroken RA, et al. The serum level of Creactive protein (CRP) is associated with cognitive performance in acute phase psychosis. BMC Psychiatry. 2016;16:60. doi:10.1186/ s12888-016-0769-x
26. Contreras-Shannon V, Heart DL, Paredes RM, et al. Clozapine-induced mitochondria alterations and inflammation in brain and insulin-responsive cells. PLoS One. 2013;8(3):e59012. doi:10.1371/journal. pone.0059012

27. Wojciak P, Remlinger-Molenda A, Rybakowski J. Stages of the clinical course of schizophrenia - staging concept. Psychiatr Pol. 2016;50(4):717-730. doi:10.12740/PP/58723

28. Radic K, Curkovic M, Bagaric D, Vilibic M, Tomic A, Zivkovic M. Ethical approach to prevention of schizophrenia - concepts and challenges. Psychiatr Danub. 2018;30(1):35-40. doi:10.24869/ psyd.2018.35

\section{Publish your work in this journal}

Neuropsychiatric Disease and Treatment is an international, peerreviewed journal of clinical therapeutics and pharmacology focusing on concise rapid reporting of clinical or pre-clinical studies on a range of neuropsychiatric and neurological disorders. This journal is indexed on PubMed Central, the 'PsycINFO' database and CAS, and is the official journal of The International Neuropsychiatric Association (INA). The manuscript management system is completely online and includes a very quick and fair peer-review system, which is all easy to use. Visit http://www.dovepress.com/testimonials.php to read real quotes from published authors. 